\title{
ANÁLISIS ESTRUCTURAL DEL EXTRACTO ETANÓLICO DEL TALLO DE Brunfelsia grandiflora D. DON Y SU EFECTO NEUROPATOLÓGICO EN EL CEREBELO DE RATA
}

\author{
Structural analysis of ethanol extract of the stem of Brunfelsia grandiflora D. Don and its \\ neuropathological effect on rat cerebellum
}

Américo J Castro L ${ }^{1}$, José R Juárez E ${ }^{1}$, Norma J Ramos $C^{1}$, José E Raez G ${ }^{2}$, Fernando O Retuerto $\mathrm{P}^{3}$, Sixto A Gonzales E ${ }^{4}$

${ }^{1}$ Instituto de Ciencias Farmacéuticas y Recursos Naturales “Juan de Dios Guevara”, Facultad de Farmacia y Bioquímica;

${ }^{2}$ Instituto de Patología de la Facultad de Medicina; ${ }^{3}$ Instituto de Investigación “Antonio Raimondi” Facultad de Ciencias Biológicas; ${ }^{4}$ Laboratorio de Toxicología de la Morgue Central de Lima.

\section{RESUMEN}

El objetivo del estudio fue elucidar la estructura del extracto etanólico del tallo de Brunfelsia grandiflora D. Don y evaluar su citotoxicidad y el efecto neuropatológico en el cerebelo de rata. Obtenido el extracto se realizó la elucidación estructural por Cromatografía de Gas /Espectrometría de Masa (CG/EM), identificándose los componentes químicos: Etil éster del ácido vainíllico, octacosano, octadecano, ácido palmítico, 7 hidroxi-6-metoxi-cumarina, linoleato de etilo y ácido linoleico. El análisis citotóxico se evaluó en embriones de Tetrapygus niger erizo de mar negro, aplicando diferentes concentraciones del extracto. La evaluación del efecto neuropatológico del extracto etanólico en el cerebelo de rata se realizó en 30 ratas albinas, cepa Holtzmann, machos, con peso corporal promedio de 2509 aproximadamente. Para el estudio del efecto neuropatológico se administraron concentraciones de 7,5 y 15 mg/mL del extracto. Se observó disminución y deformación de las neuronas con protoplasma desorientado, balonadas, vacuoladas y necrosadas; con núcleos grandes y deformes y otras sin núcleo, anisocitosis y poiquilocitosis. Se concluye que el extracto etanólico del tallo de la especie estudiada, tiene efecto neuropatológico en el cerebelo de rata. Esta investigación se basa en el estudio de la droga y su efecto neuropatológico que afecta el núcleo cerebelar profundo y la coordinación motriz del material biológico utilizado.

Palabras clave: Brunfelsia grandiflora D. Don, citotoxicidad, neuropatológico, Cromatografía de Gases / Espectrometría de Masas (CG/EM).

\section{SUMMARY}

The objective of the study was to elucidate the structure of the etanólic extract of the stem of Brunfelsia grandiflora D. Don and to evaluate its citotoxicity and the neuropathological effect on rat cerebellum. Obtained the extract the structural elucidation was made by Gas Chromatography/was made /Espectrometría of Masa (CG/EM), identifying the chemical components: Etil ester of vainíllic, octacosano, octadecan acid, palmític acid, 7 hidroxi-6-metoxi-cumarin, linoleate of ethyl and linoleic acid. The cytotoxic analysis was evaluated in embryos of Tetrapygus Niger black sea urchin, applying different concentrations of the extract. The evaluation of the neuropatológic effect of the etanólic extract in the rat cerebellum was made in 30 albino rats, Holtzmann strain, male stock, with corporal weight average of 2509 approximately. For the study of the neuropatológic effect were administered concentrations of 7,5 and $15 \mathrm{mg} / \mathrm{mL}$ of the extract. Were observed diminution and deformation of the neurons with protoplasm disoriented, vacuolated and necrotic; with great nuclei and deformed and others without nucleus, anisocitosis and poiquilocitosis. Was concluded that the etanólic extract of the stem of the studied species, has neuropatológic effect in the rat cerebellum. This investigation was based on the study of the drug and its neuropatológic effect that affects to deep cerebelar nucleus and motor coordination of the bacteriological agents used.

Keywords: Brunfelsia grandiflora D. Don, cytotoxicity, neuropathological, Gas Chromatography / Mass Spectrometry (GC / MS).

\section{INTRODUCCIÓN}

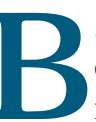

runfelsia grandiflora D. Don, conocida como "Chiric sanango", es una planta nativa de la selva amazónica del Perú. Esta especie crece en Sudamérica desde Venezuela hasta Bolivia. En el Perú se distribuye en la selva amazónica hasta una altitud de 200 metros en los departamentos de Loreto, San Martín y Cuzco, comprendiendo la Amazonía baja y alta.

En la medicina popular se le atribuye efecto cicatrizante en lesiones de la piel ${ }^{(1)}$. Los nativos y pobladores de la sierra y selva del Perú lo utilizan para la 
cicatrización de heridas producidas por Leishmaniasis (4). Este arbusto es utilizado como aditivo para preparar "Ayahuasca" entre los Jíbaros y Kofanes, y se sospecha que lo usan por su poderoso efecto narcótico ${ }^{(2,3)}$.

El extracto se obtuvo por maceración y el análisis de su composición química se realizó por Cromatografía de Gas / Espectrometría de Masa (CG/EM). La actividad citotóxica se evaluó en el desarrollo embrionario de Tetrapygus niger y el efecto neuropatológico del extracto, en cerebelo de rata.

La investigación realizada sobre la composición química del tallo es escasa, al igual que los estudios sobre su actividad citotóxica y efecto neuropatológico en las células de Purkinje del cerebelo de rata, por lo que el objetivo del presente estudio fue elucidar la estructura del extracto, evaluar su citotoxicidad y efecto neuropatológico, así como las características de dosis-respuesta y el daño neuronal en el cerebelo de rata en un intervalo de siete días.

\section{MATERIALES Y MÉTODOS}

La investigación es de tipo experimental, analítica, prospectiva y longitudinal. La especie vegetal fue colectada en el distrito de Contamana, provincia de Iquitos, en el departamento de Loreto, a 200 metros de altitud, realizándose su clasificación taxonómica en el Museo de Historia Natural de la Universidad Nacional Mayor de San Marcos, según la siguiente posición sistemática de acuerdo a Cronquist (1988):

$\begin{array}{ll}\text { División } & \text { : Magnoliophyta } \\ \text { Clase } & \text { : Magnoliopsida } \\ \text { Orden } & : \text { Serophulariales } \\ \text { Familia } & : \text { Solanaceae } \\ \text { Género } & : \text { Brunfelsia } \\ \text { Especie } & : \text { Brunfelsia grandiflora D. Don } \\ \text { Nombre vulgar } & : \text { "Chiric sanango" }\end{array}$

La obtención del extracto se realizó por el método de maceración alcohólica de o1 kg de tallo trozado con tres litros de etanol $96^{\circ}$ durante siete días con agitación diaria. Posteriormente, se filtro y evaporó a sequedad en estufa de aire circulante a $40^{\circ} \mathrm{C}$, obteniéndose $35 \mathrm{~g}$ de extracto. El análisis estructural de la composición química del extracto, se efectuó por Cromatografía de Gas / Espectrometría de Masas (CG/EM) ${ }^{(5,6)}$.

\section{Determinación de la actividad citotóxica}

El bioensayo serealizóenel desarrolloembrionario de Tetrapygus niger, erizo de mar negro, a las 48 horas de fecundación in vitro. Se trabajó con 20 muestras de erizo de mar negro colectadas en la playa de Ancón, ubicada a $43 \mathrm{Km}$ al norte de Lima, y trasladadas en agua de mar entre 10 y $15^{\circ} \mathrm{C}$. Este proceso comprendió la obtención y diferenciación de los gametos. Los óvulos se suspendieron en solución de $500 \mathrm{~mL}$ de agua de mar y en oxigenación continua. La fertilización se logró mezclando suavemente los espermatozoides y observando al microscopio las muestras fecundadas en láminas portaobjetos. Se tomaron muestras cada dos horas hasta observar cuál era la dilución en la que se producía el mayor porcentaje de células muertas durante el desarrollo embrionario de Tetrapygus niger. Las concentraciones del extracto etanólico ensayadas fueron de 5,$2 ; 7 ; 5 ; 10,5 ; 15 ; 15,75 ; 21 ; 22,5$ y $30 \mathrm{mg} / \mathrm{mL}$.

\section{Efecto neuropatológico}

La investigación farmacológica para la evaluación del efecto neuropatológico del extracto etanólico en el cerebelo de rata, se realizó en 30 ratas albinas cepa Holtzmann, machos de 250 g aproximadamente de peso corporal. Las ratas fueron obtenidas en el Instituto Nacional de Salud - Centro Nacional de Productos Biológicos - Chorrillos.

Los animales estuvieron bajo condiciones de manejo y sanidad que articulaban conceptos técnicos, éticos, de calidad, de validez y competitividad científica. Fueron distribuidos en cinco grupos. Se administró al primer grupo $5 \mathrm{~mL}$ de suero fisiológico y a los grupos 2, 3, 4 y 5_se les dio 3,5; 4,5; 3,5 y 4 mL de droga en concentraciones de 7,$5 ; 15 ; 22,5$ y $33 \mathrm{mg} / \mathrm{mL}$, respectivamente, durante siete días. Posteriormente los animales fueron sacrificados por inhalación de cloroformo, procediéndose a extraer el cerebelo para el análisis histopatológico.

\section{RESULTADOS}

La maceración etanólica del tallo de Brunfelsia grandiflora D. Don dio un rendimiento de $35 \mathrm{~g}$ de extracto de color oscuro, sabor amargo y de reacción ligeramente ácida. En las figuras 1, 2 y tabla 1 se presentan los resultados de la elucidación estructural y la composición química del extracto del tallo realizado por Cromatografía de Gas / Espectrometría de Masa (CG/EM).

La determinación de la actividad citotóxica según los niveles de concentración en $\mathrm{mg} / \mathrm{mL}$ del extracto etanólico del tallo, se presentan en las tablas 2, 3 y 4. Asimismo, en la tabla 5 se presentan los resultados desarrollados a las tres horas de iniciado el proceso de fecundación de los gametos de Tetrapygus niger. 


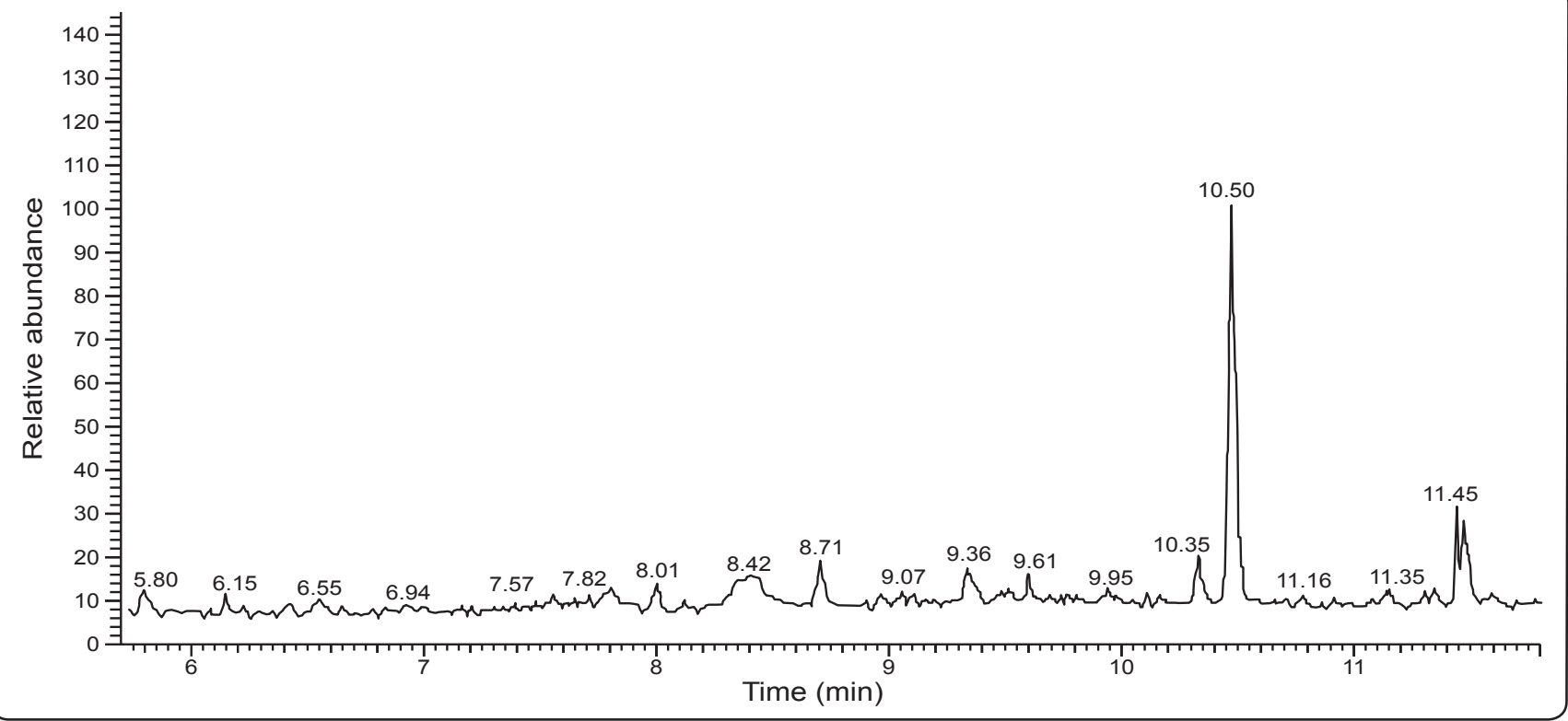

Figura 1. Cromatografía de Gas del extracto etanólico del tallo de Brunfelsia grandiflora D. Don

La evaluación del extracto, aplicado en concentraciones de 5,25; 7,5; 10,5; 15; 15,75 y $21 \mathrm{mg} / \mathrm{mL}$ demostró efecto citotóxico (figuras 3, 4, 5, 6 y 7).

A concentraciones de 22,5 y $30 \mathrm{mg} / \mathrm{mL}$ manifestó mutación y aberraciones celulares en el desarrollo embrionario in vitro (figuras: $8,9,10$ y 11 ).

Respecto al efecto neuropatológico del extracto etanólico del tallo de Brunfelsia grandiflora D. Don, en el grupo control no presentó alteraciones anátomo-histopatológicas en las neuronas de

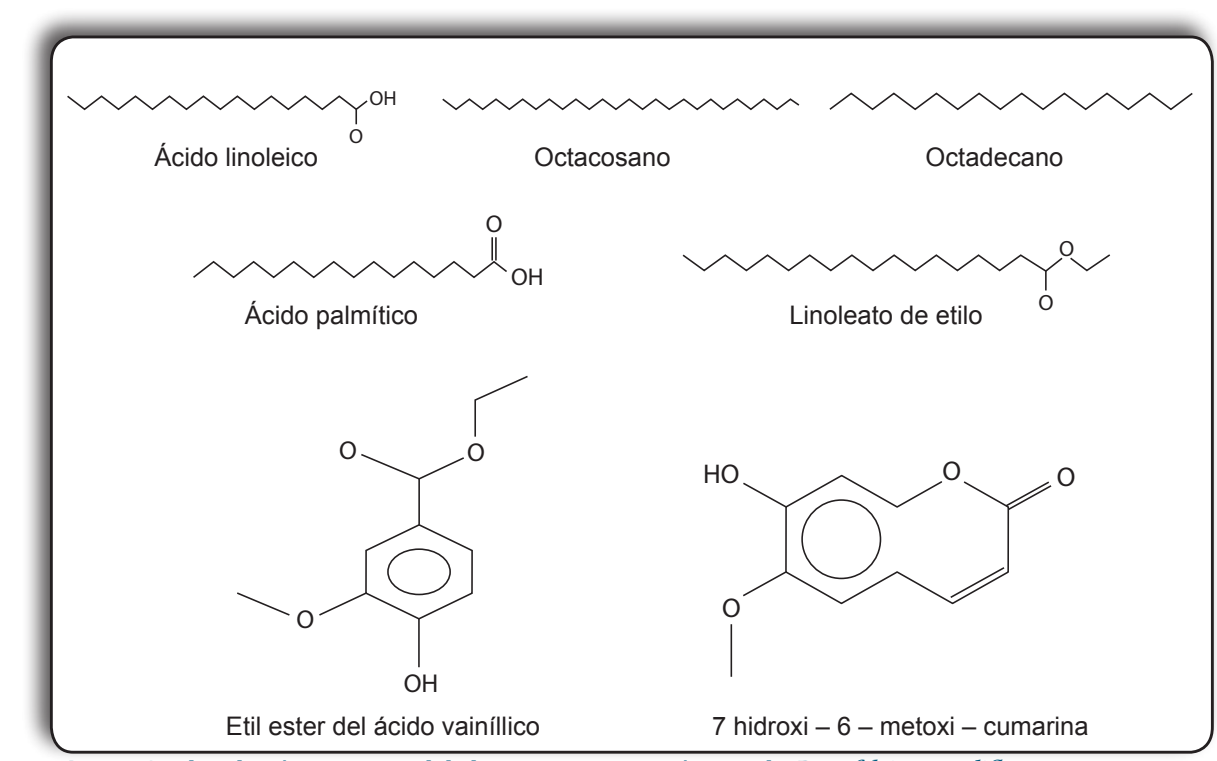

Figura 2. Elucidación estructural de los compuestos químicos de Brunfelsia grandiflora D. Don
Purkinje, localizadas en la corteza cerebelar_(figura 12).

Con la administración de 7,5 y $15 \mathrm{mg} / \mathrm{mL}$ del extracto, se observó disminución y deformación de las neuronas con protoplasma desorientado, células balonadas, vacuoladas y necrosadas, con núcleos grandes y deformes y otras sin núcleos, anisocitosis y poiquilocitosis (figuras: 13, 14, 15, 16 y 17).

A las concentraciones de 22,5 y $30 \mathrm{mg} / \mathrm{mL}$ de extracto, se observaron células de Purkinje en estado edematoso y muerte celular con células gliales disminuidas, estas últimas desempeñan la función de soporte de las neuronas (figuras: 19 y 20).

\section{DISCUSIÓN}

Existen principios activos naturales que tienen acción alucinógena y están presentes en especies de nuestra Amazonía. $\mathrm{Su}$ ingestión en complicadas ceremonias o ritos, provoca variaciones en el estado de ánimo, causando desde miedo profundo hasta franca euforia, desorientación, turbación de la memoria y afectación de los receptores celulares del 


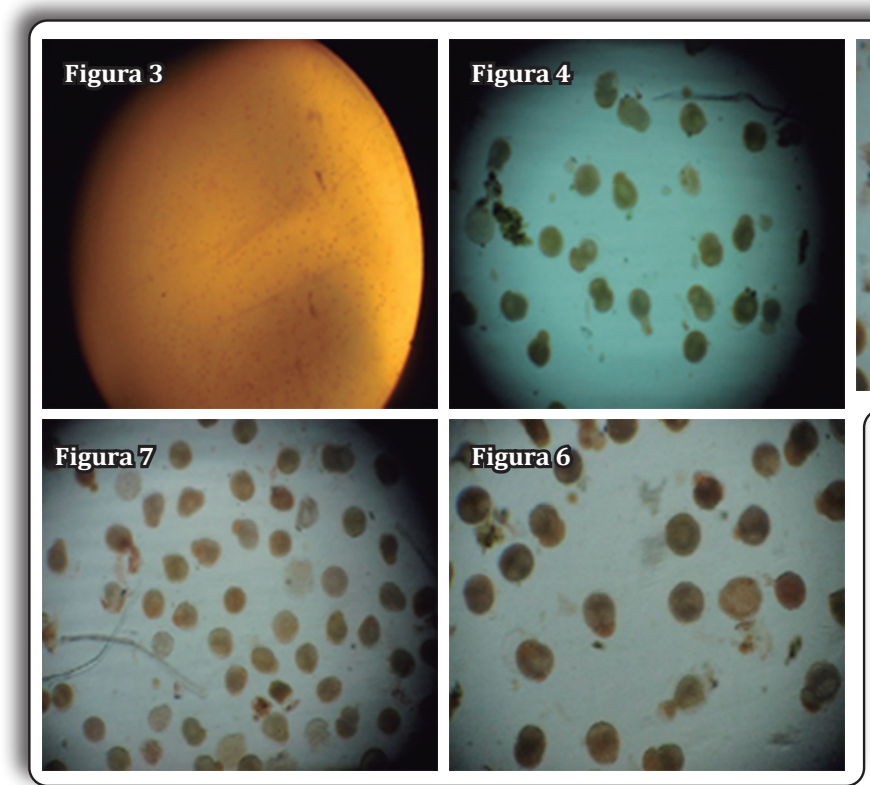

Presencia de citotoxicidad en el desarrollo embrionario in vitro de Tetrapygus niger, HE 400X.

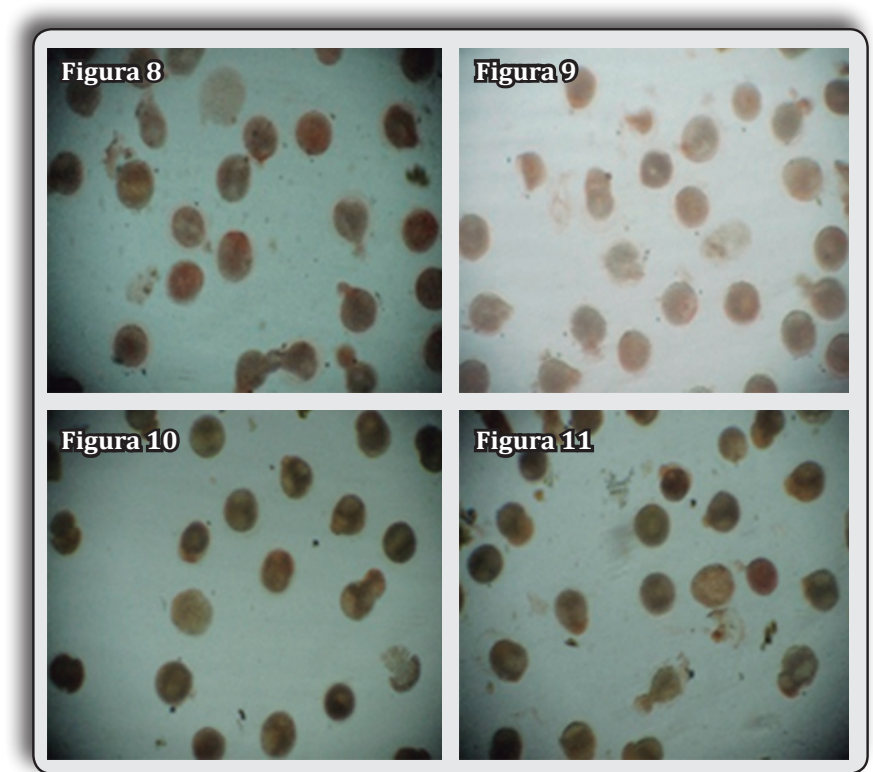

Presencia de mutación y aberración celular en el desarrollo embrionario in vitro de Tetrapygus niger, HE $400 X$.

Tabla 1. Composición química del extracto etanólico de Brunfelsia grandiflora D. Don determinado por Cromatografía de Gas/ Espectrometría de Masa (CG/EM).

\begin{tabular}{lc}
\hline \multicolumn{1}{c}{ Composición química } & $\begin{array}{c}\text { Tiempo de Retención } \\
\text { (TR) (minutos) }\end{array}$ \\
\hline Etil ester del ácido vaníllico & 8,01 \\
Octacosano & 8,71 \\
Octadecano & 9,36 \\
Ácido palmítico & 10,35 \\
7-hidroxi-6-metoxi-cumarina & 10,50 \\
Linoleato de etilo & 11,45 \\
Ácido linoleico & 11,49 \\
\hline
\end{tabular}

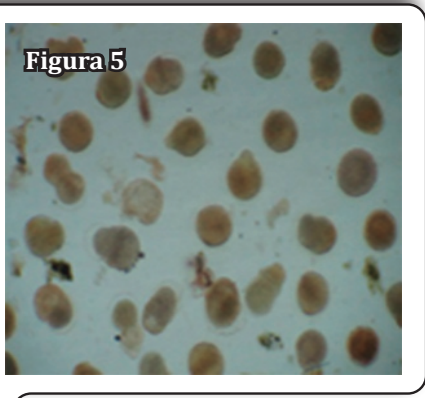

organismo humano; en dosis excesivas pueden producir la muerte.

Al estudiar la composición química, el análisis por Cromatografía de Gas / Espectrometría de Masa (CG/EM), del extracto etanólico del tallo de Brunfelsia grandiflora D. Don identificó, entre otros componentes, al principio activo 7-hidroxi-6-metoxicumarina, conocido también como escopoletina. Hallazgo que confirma lo reportado en otros trabajos sobre su composición química donde se relaciona a este compuesto con actividad leishmanicida ${ }^{(7,8)}$. Al respecto, Fuchino et

Tabla 2. Concentración del extracto en $\mathrm{mg} / \mathrm{mL}$.

\begin{tabular}{cc}
$\mathbf{N}^{\circ}$ Extracto & Concentración $(\mathrm{mg} / \mathrm{mL})$ \\
\hline C1 & 7,5 \\
C2 & 15,0 \\
C3 & 22,5 \\
C4 & 30,0 \\
\hline
\end{tabular}

Tabla 3. Primera batería: Viales con volumen de extracto, huevos fecundados y agua de mar.

\begin{tabular}{ccccc}
\hline $\mathbf{N}^{\circ}$ de Vial & $\begin{array}{c}\text { mg de } \\
\text { extracto } \\
\text { en 7 mL }\end{array}$ & $\begin{array}{c}\text { Volumen } \\
\text { de huevos } \\
\text { fecundados }\end{array}$ & $\begin{array}{c}\text { mL de } \\
\text { Agua de } \\
\text { mar }\end{array}$ & $\begin{array}{c}\text { Concentración } \\
\text { final (mg/mL) }\end{array}$ \\
\hline A0 (control) & - & 2 & 1 & 0 \\
A1 & 52,5 & 2 & 1 & 5,25 \\
A2 & 105 & 2 & 1 & 10,5 \\
A3 & 157,5 & 2 & 1 & 15,75 \\
A4 & 210 & 2 & 1 & 21,0 \\
\hline
\end{tabular}

Tabla 4. Segunda batería: Viales con miligramos de extracto, huevos fecundados y agua de mar.

\begin{tabular}{ccccc}
\hline $\mathbf{N}^{\circ}$ de Vial & $\begin{array}{c}\text { mL de } \\
\text { extracto } \\
\text { en 7 mL }\end{array}$ & $\begin{array}{c}\text { Volumen } \\
\text { de huevos } \\
\text { fecundados }\end{array}$ & $\begin{array}{c}\text { mL de } \\
\text { agua de } \\
\text { mar }\end{array}$ & $\begin{array}{c}\text { Concentración } \\
\text { final (mg/mL) }\end{array}$ \\
\hline R0 (control) & - & 2 & 1 & 0 \\
R1 & 75 & 2 & 1 & 7,5 \\
& 150 & 2 & 1 & 15,0 \\
R3 & 225 & 2 & 1 & 22,5 \\
R4 & 300 & 2 & 1 & 30 \\
\hline
\end{tabular}

Tabla 5. Resultados a las tres horas de fecundación de los gametos de Tetrapygus niger.

\begin{tabular}{cccc}
$\mathbf{N}^{\circ}$ Vial & $\begin{array}{c}\text { Extracto } \\
\text { Etanólico (mg) }\end{array}$ & Citotoxicidad & Observación \\
\hline Control & 0 & - & Desarrollo normal \\
1 (A1) & 5,25 & + & Citotóxico \\
2 (R1) & 7,5 & + & Citotóxico \\
3 (A2) & 10,5 & + & Citotóxico \\
4 (R2) & 15,0 & + & Citotóxico \\
5 (A3) & 15,75 & ++ & Citotóxico \\
6 (A4) & 21,0 & ++ & Citotóxico \\
7 (R3) & 22,5 & +++ & Mutación \\
8 (R4) & 30,0 & +++ & Mutagénesis y \\
\hline
\end{tabular}

Leyenda: (-) Negativo, (+) Positivo, (++) Abundante, (+++) Muy abundante. 
al., aislaron del extracto metanólico de las hojas de esta especie vegetal, una saponina con potente actividad leishmanicida in vitro contra Leishmania major, y también escopoletina, al que atribuyeron moderada actividad leishmanicida (9). Otra investigación realizada en el extracto metanólico de esta planta indica la presencia de componentes químicos de naturaleza nitrogenada de tipo alcaloide indólico ${ }^{(10)}$.

Las cumarinas son lactonas de los ácidos o-hidroxicinámicos y se encuentran difundidos en las familias Solanaceae, Apiaceae, Rutaceae y Asteraceae, entre otras. La concentración de sus principios activos es mayor en raíces, semillas y frutos ${ }^{(11)}$.

Con la aplicación del bioensayo de citotoxicidad en embriones de Tetrapygus niger erizo de mar negro; se pudo determinar la actividad del extracto etanólico del tallo de Brunfelsia glandiflora

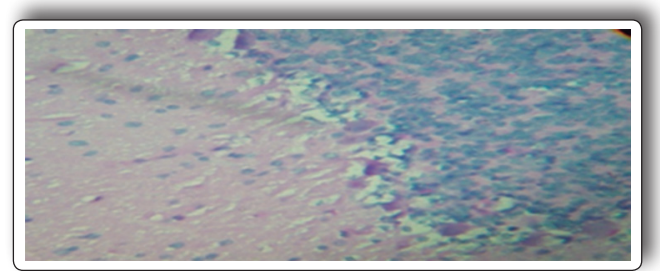

Figura 12. Células de Purkinje coloreadas y espaciadas, LFB 400X.

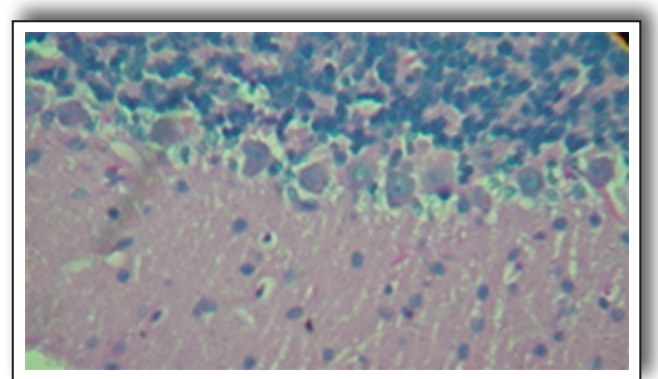

Figura 13. Células de Purkinje dañadas y deformadas, con anisocitosis y poiquilocitosis, LFB 400X.

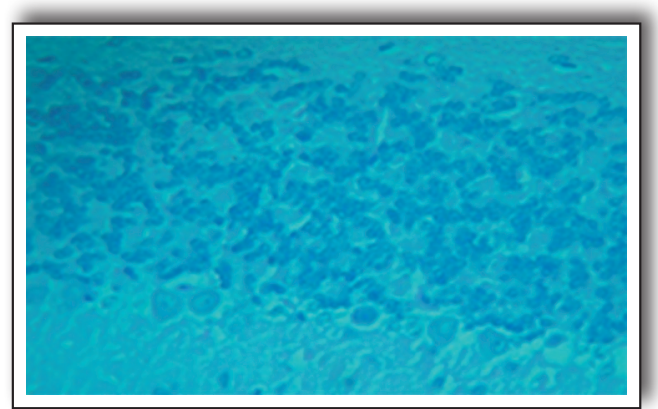

Figura 14. Células de Purkinje con núcleos muy grandes, LFB 400X.

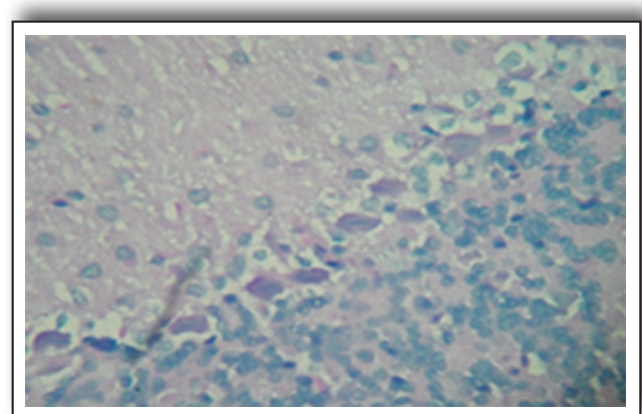

Figura 15. No hay presencia de células gliales, LFB 400X.
D. Don, a través de efectos citotóxicos, mutaciones y aberraciones celulares en el desarrollo embrionario in vitro de Tetrapygus niger ${ }^{(12,13)}$.

Las cumarinas han sido utilizadas como corrector de olor y sabor en productos farmacéuticos. En experimentos con animales se han observado lesiones hepáticas y propiedades cancerígenas. La cumarina condensada dicumarol inhibe la formación de protrombina, proconvertina y del factor Stuart-Power

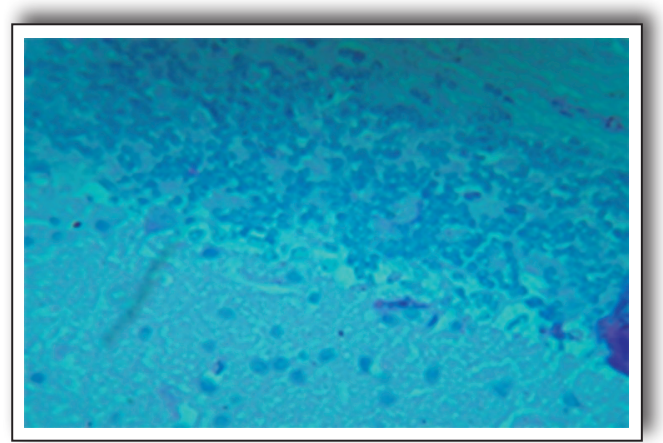

Figura 16. Células de Purkinje sin núcleo, LFB 400X.

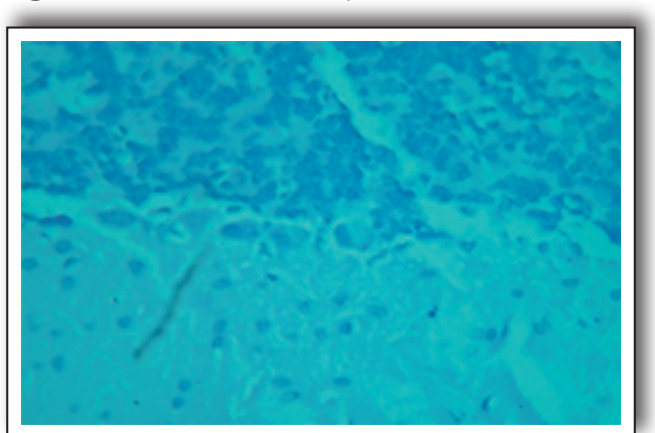

Figura 17. Células de Purkinje deformadas, LFB 400X.

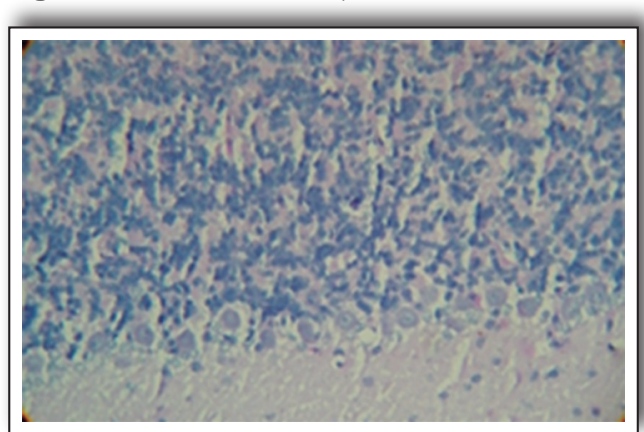

Figura 19. Células gliales disminuidas, LFB 400X.

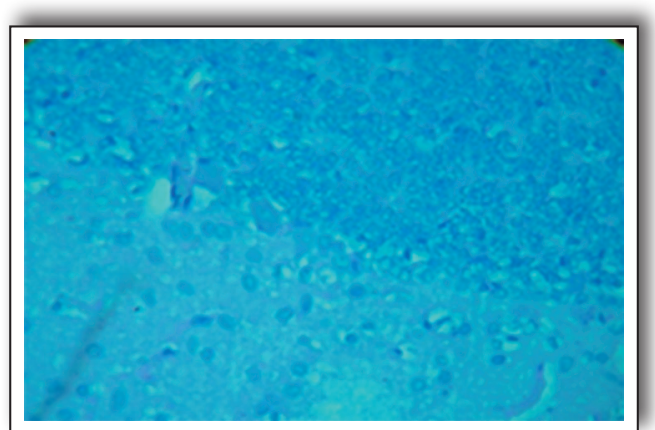

Figura 20. Células de Pukinje desordenadas, en estado edematoso y apoptosis, LFB 400X.

piendo la

capacidad

de coagulación de la sangre por desalojo de la vitamina $\mathrm{K}^{(14)}$.

Brunfelsia glandiflora D. Don, se utiliza como embriagante en nuestra amazonía y como aditivo de la 
ayahuasca en Ecuador y Perú. También se ha reportado el aislamiento de) una sustancia convulsivante, la brunfelsamidinia ${ }^{(15)}$.

El principal efecto secundario no deseado del escopoletina es el de incrementar el tiempo de sangrado (16). Los derivados de la cumarina son utilizados como anticoagulantes para el tratamiento de trastornos en los que hay excesiva o indeaseada coagulación, tales como tromboflebitis, embolismo pulmonar y ciertas condiciones cardíacas. También son utilizados como rodenticidas, debido a la propiedad de causar hemorragias nasales.

En el estudio realizado sobre los grupos 1 y 2 de ratas tratadas con el extracto etanólico en concentraciones de 7,5 y $15 \mathrm{mg} / \mathrm{mL}$ del extracto, respectivamente, se pudo observar, en los animales, parcial caída del pelaje y movimientos descoordinados leves. En los grupos 3 y 4 a las concentraciones de 22,5 y $30 \mathrm{mg} / \mathrm{mL}$ del extracto, respectivamente, se observó aumento en la caída del pelaje, alteración en los movimientos, desequilibrio, dilatación de los ojos, temblores, hemorragia nasal, convulsiones, llegandoen tres casosa causar la muerte previa contracción muscular, temblor y brincos descoordinados y chillidos. Estas características observadas nos llevan a coincidir con los efectos de sobredosis que tiene el escopoletin sobre las células de Purkinje manifestando estremecimientos intencionales, hiperactividad, falta de reflejos, e inhabilidad general para determinar el espacio, distancia y descoordinación motriz.

\section{CONCLUSIONES}

- En el extracto etanólico del tallo de Brunfelsia grandiflora D. Don se elucidaron los siguientes componentes químicos: Etil ester del ácido vaníllico, octacosano, octadecano, ácido palmítico, 7-hidroxi-6-metoxi-cumarina, linoleato de etilo y ácido linoleico.

- En la determinación de la actividad toxicológica del extracto etanólico en el desarrollo embrionario de Tetrapygus niger, erizo de mar negro, se encontró efecto citotóxico en concentraciones de 5,$25 ; 7,5 ; 10,5 ; 15 ; 15,75$ y 21 $\mathrm{mg} / \mathrm{mL}$, mientras que a concentraciones de 22,5 y $30 \mathrm{mg} / \mathrm{mL}$, se observó mutación y aberración celular.

- El extracto etanólico del tallo de Brunfelsia grandiflora D. Don administrado a las concentraciones de 7,5 y $15 \mathrm{mg} / \mathrm{mL}$, alteró la citomorfología de las neuronas de Purkinje localizadas en la corteza cerebelosa de ratas causando células deformes, necrosadas, con núcleos grandes y células sin núcleos, anisocitosis y poiquilocitosis. En las concentraciones de 22,5 y $30 \mathrm{mg} / \mathrm{mL}$ del extracto se observaron células de Purkinje en estado edematoso y muerte celular, determinándose así el efecto neuropatológico del extracto en cerebelo de rata.

\section{REFERENCIAS BIBLIOGRÁFICAS}

1. Brack EA. Diccionario enciclopédico de plantas útiles en el Perú, Cusco. Centro Bartolomé de las Casas. Cuzco, 1999. p. 82.

2. Lloyd HA, Fales HM, Goldman ME, Jerina DM, Plowman T, Schultes RE. Brunfelsamidine: a novel convulsant from the medicinal plant Brunfelsia grandiflora. Tetrahedron Lett 1985; 26(22): 2623-4.

3. Soukup J. Vocabulario de los nombres vulgares de la flora peruana y catálogo de los géneros. Editorial Salesiana. Lima, 1987. p. 92.

4. Muñoz AM, Pareja B. Plantas medicinales empleadas en el tratamiento de la leishmaniasis. Dermofarmacia. Folia Dermatol 2003; 14 (3): 10-4.

5. De Hoffmann E, Stroobant V. Mass spectrometry, principles and applications. $3^{\text {th }}$ ed. John Wiley \& Sons. West Sussex, 2007. p. 217-32.

6. Ekman $\mathrm{R}$ et al. Mass spectrometry: instrumentation, interpretation and applications. John Wiley \& Sons. New Yersey, 2009. p. 121-7.

7. Torres-Santos EC, Lopes D, Oliveira RR, Carauta JP, Falcao CA, Kaplan MA, et al. Antileishmanial activity of isolated triterpenoids from Pourouma guianensis. Phytomedicine 2004; 11(2-3): 114-20.

8. Del Rayo M, Phillipson JD, Croft SL, Kirby GC, Warhurst DC, Solis PN. Terpenoides de Guarea rhopalocarpa. Phytochemistry. 2001; 56(2):203-10.

9. Fuchino H, Sekita S, Mori K, Kawahara N, Satake M, Kiuchi F. A new leishmanicidal saponin from Brunfelsia grandiflora. Chem Pharm Bull (Tokyo). 2008; 56(1): 93-6.

10. Ramos CN. Composición química, actividad antioxidante in vitro y evaluación cicatrizante in vivo del extracto metanólico de corteza de Brunfelsia grandiflora D. Don "chiric sanango". [Tesis Doctoral] Universidad Nacional Mayor de San Marcos. Lima, 2012. p. 43.

11. Murray RDH.Naturally Occurring Plant Coumarins. Fortschr Chem Org Naturst 1997; 72: 1-119.

12. HoseJE. Potential uses of sea urchin embryos for identifying toxic chemicals: description of a bioassay incorporating cytologic, cytogenetic and embryologic endpoints. Journal of Applied Toxicology 1985; 5(4):245-54.

13. Castro LA, Juárez EJ, Ramos CN, Retuerto PF, Gonzales ES. Elucidación estructural del aceite esencial de 
Ruta graveolens L. ruda, actividad antioxidante y bioensayo de citotoxicidad. Ciencia e Investigación. 2011; 14(1):25-8.

14. Gros EG, Pomillo AB, Seldes AM, Burton G. Introducción al estudio de los productos naturales. Facultad de Ciencias Exactas y Naturales. Universidad de Buenos Aires. Secretaría General de la Organización de los Estados Americanos. Programa regional de desarrollo Científico y Tecnológico. Washington DC, 1985.

15. Obasi SC et al. Effects of single oral doses of scopoletin and aflatoxin $\mathrm{B} 1$ on the clotting time, serum cholesterol and phospholipid levels of chicks._J Physiol Pharmacol Indian. 1994; 38(2): 89-94.

Manuscrito recibido el: 07/o1/2013

Aceptado para su publicación el: 27/03/2013

\section{Correspondencia:}

Nombre: Dr. Américo J. Castro Luna

Dirección: Jr. Puno 1002 Lima 1-Perú

e-mail: caslasha3@hotmail.com 